\title{
The effects of acidic functional groups and particle size of biochar on Cd adsorption from aqueous solutions
}

\begin{abstract}
The removal of $\mathrm{Cd}$ from the wastewater is necessary because of its harmful health effects. The practice of using biochar as a low-cost adsorbent for heavy metals removal from water bodies is common. However, the effects of total acidic functional groups and particle size class on heavy metals removal by biochar are not studied well. Therefore, this study was undertaken with the objective of determining the effects of total acidic functional groups and particle size class on $\mathrm{Cd}$ adsorption from aqueous solution by an empty fruit bunch biochar (EFBB) and a rice husk biochar (RHB). The results showed that there was no significant difference in the carbon content between the EFBB and RHB. However, higher quantity of total acidic functional groups was found in the EFBB compared to the RHB. The total acidic functional groups of EFBB were higher than of the RHB for the same particle size class. In contrast, the surface area of RHB was higher than the EFBB for the same size class. The Langmuir's maximum adsorption capacity (Qmax) of EFBB was higher than RHB when compared at each particle size class. Significant correlations were observed between Qmax and the total acidic functional groups of both biochars. There were significant correlations between Qmax and the cation exchange capacity (CEC) as well. However, the correlations were non-significant between Qmax and particle size, surface area and pore volume of both biochars. It can be concluded that only the total acidic functional groups and the CEC were influential in determining the adsorption capacities of both EFBB and RHB for Cd adsorption.
\end{abstract}

Keyword: Surface area; Cation exchange capacity; Empty fruit bunch biochar; Rice husk biochar 